Orthopäde 2011 · 40:281

DOI 10.1007/s00132-010-1661-x

Online publiziert: 18. Februar 2011

(c) Springer-Verlag 2011

F. Gohlke

Klinik für Schulterchirurgie, Bad Neustadt/Saale

\title{
Das steife Ellenbogengelenk
}

Sehr geehrte Leserinnen und Leser,

obwohl sich die Behandlung von Verletzungen und Erkrankungen des Ellenbogengelenks in den letzten Jahren zunehmend etabliert hat, gibt es in Deutschland im Vergleich zum angloamerikanischen Raum immer noch wenig orthopädische Chirurgen, die sich schwerpunktmäßig mit diesem Gelenk beschäftigen.

Das mag daran liegen, dass dieses Gebiet immer noch im berufspolitischen Spannungsfeld zwischen Traumatologen und eher konservativ ausgerichteten Orthopäden liegt und derzeit entweder der Hand- oder aber Schulterchirurgie zugeschlagen wird. Global gesehen, befassten sich in den USA und Asien die Schulterchirurgen zunächst nur am Rande mit diesem Gelenk, während in Europa unter dem dominierenden Einfluss der wissenschaftlichen Dachgesellschaft SECEC seit den 8oer Jahren das Interesse für beide Gelenke, die Schulter als auch den Ellenbogen, sowohl in der Grundlagenforschung als auch auf klinischem Gebiet intensiv gefördert wurde. Seit ihrer Gründung im Jahre 1994 hat sich aber auch in Deutschland die DVSE (Deutsche Vereinigung für Schulter- und Ellenbogenchirurgie) zum Ziel gesetzt, die klinischen und wissenschaftlichen Aktivitäten auf diesem Gebiet zu unterstützen.

\section{(2) Der Trend zum „Upper Extremity Surgeon" ist in der jüngeren Generation unverkennbar}

Da der Trend zum „Upper Extremity Surgeon" in der jüngeren Generation unverkennbar ist, wird es Zeit, sich mit den Problemen des Ellenbogengelenks schwerpunktmäßig näher zu beschäftigen. Die vorliegende Ausgabe befasst sich gleichsam als Einstieg zu einer Fortbildungsreihe mit einer für diese Region durchaus charakteristischen Entität, der Gelenksteife, die in unterschiedlicher Ausprägung sowohl als Folge traumatischer Einwirkungen als auch von Fehlbildungen oder degenerativen Erkrankungen entstehen kann.

Es kommen Autoren aus mehreren Ländern zu Wort, die sich mit den verschiedenen Aspekten dieser Gelenkproblematik bei durchaus unterschiedlichen Ansichten zu den möglichen Therapieoptionen äußern. Die Palette reicht von der konservativen, am Schmerz orientierten Behandlung bis hin zu hochspezialisierten operativen Verfahren wie der Distraktionsarthrolyse.

Damit bietet sich dem Leser die Möglichkeit zur Entwicklung einer differenzierten Betrachtungsweise, die je nach Schweregrad, Ätiologie und pathologischem Substrat ein auch an den individuellen Ansprüchen des Patienten orientiertes Vorgehen entwickelt.

Ihr
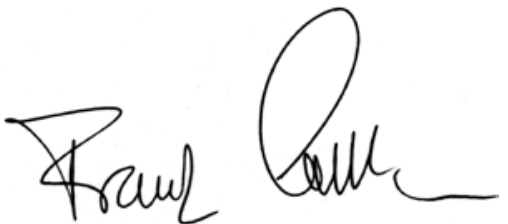

F. Gohlke

\section{Korrespondenzadresse \\ Prof. Dr. F. Gohlke}

Klinik für Schulterchirurgie

Salzburger Leite 1, 97616 Bad Neustadt/Saale frank.gohlke@schulterchirurgiebad-neustadt.de

Interessenkonflikt. Der korrespondierende Autor gibt an, dass kein Interessenkonflikt besteht. 